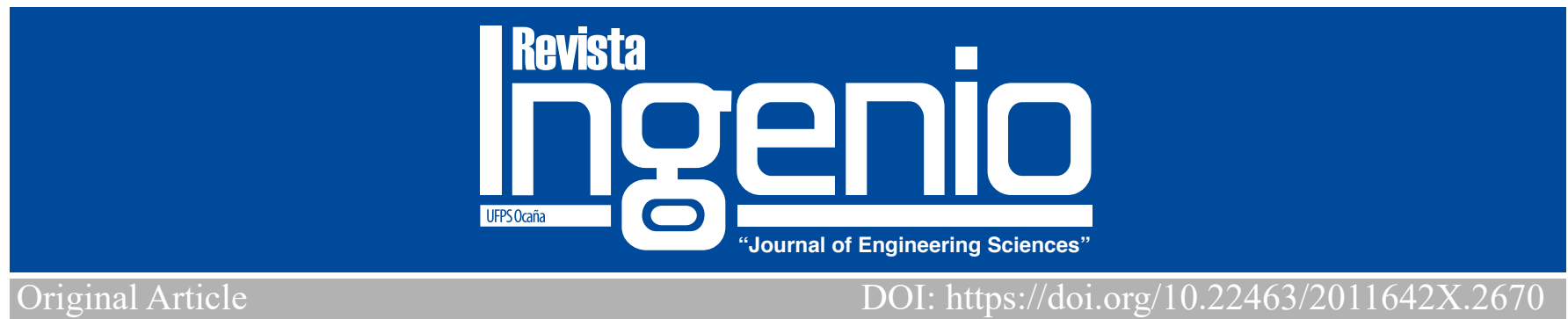

\title{
Evaluación experimental de esfuerzos usando la correlación de imagen digital
}

\author{
Experimental stress evaluation using digital image correlation

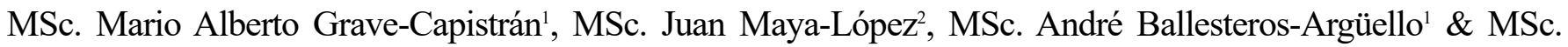 \\ Ricardo Andrés García-León ${ }^{1-3^{*}}$
}

${ }^{1}$ Instituto Politécnico Nacional. SEPI-ESIME, Zacatenco. CDMX, México, Orcid: https://orcid.org/0000-0003-0911-4963, https://orcid.org/0000-0001-8658-7786 \& https://orcid.org/0000-0002-2734-1425. Email:mariograveca@gmail.com, aballesteros_90@hotmail.com,raqarcial@gmail.com*

${ }^{2}$ Instituto Tecnológico Superior de Poza Rica, Poza Rica, Veracruz, México. Orcid: https://orcid.org/0000-0002-0146-1832.Email: juanmaya1914@gmail.com,

${ }^{3}$ Universidad Francisco de Paula Santander, Ocaña, Colombia.

Como citar: M. A. Grave, J. Maya, A. Ballesteros \& R. A. García, "Evaluación experimental de esfuerzos usando la correlación de imagen digital", Revista Ingenio, vol. 18 (1), pp. 48-53, 2021

\begin{tabular}{|c|c|}
\hline & RESUMEN \\
\hline Palabras claves: & $\begin{array}{l}\text { En este trabajo se llevó a cabo el uso de herramientas computacionales para predicción de falla en el material PLA (Ácido Poli } \\
\text { Láctico) obtenido mediante impresión 3D. Las evaluaciones en muestras en fase de prototipo suelen tener cambios hasta conseguir }\end{array}$ \\
\hline $\begin{array}{l}\text { Deformación, esfuerzos, } \\
\text { imágenes, simulación } \\
\text { numérica. }\end{array}$ & $\begin{array}{l}\text { un diseño a detalle, estos cambios provienen de ensayos que se realizan para comprobar su funcionamiento. La implementación de } \\
\text { simulaciones numéricas y paqueterías de predicción ha revolucionado la forma en que se modifican muestras hasta alcanzar una } \\
\text { manufactura óptima para su aplicación en diferentes sectores industriales. La técnica de moteado en muestras y empleando el método } \\
\text { de correlación de imagen digital (DIC), se ha establecido como solución para inspección en muestras que son sometidas a ensayos } \\
\text { de esfuerzos obteniendo buenos resultados. Los desplazamientos analizados en la muestra son verificados en relación al esfuerzo de } \\
\text { compresión aplicado obteniendo un máximo de desplazamiento encontrado por medio de los patrones en la muestra de hasta } 15.0 \% \\
\text { aplicando una fuerza de } 1 \mathrm{~N} \text { en } 15.725 \mathrm{~s} \text {, estos resultados se validaron enpleando la paquetería para simulación y análisis utilizando } \\
\text { el software solidworks. }\end{array}$ \\
\hline
\end{tabular}

\section{ABSTRACT}

Key words:

Deformation, stress, images, numerical analysis.
In this work, the use of computational tools for the prediction of failure in the PLA(Poly Lactic Acid) material obtained by 3D printing was carried out. The evaluations in samples in the prototype stage usually have changed until a detailed design is achieved, these changes come from tests that are carried out to verify their operation. The implementation of numerical simulations and prediction packages has revolutionized the way in which parts are modified until reaching optimal manufacturing for application in different industrial sectors. The speckled technique in samples and using the digital image correlation method (DIC), has been established as a solution for inspection in samples that are subjected to stress tests obtaining excellent results. The displacements analyzed in the sample are verified in relation to the applied compression stress, obtaining a maximum displacement found by means of the patterns in the sample of up to $15.0 \%$ applying a force of $1 \mathrm{~N}$ in $15.725 \mathrm{~s}$, These results we're validat es employing the package for simulation and using solidworks software.

\section{Introducción}

El ácido poliláctico (PLA) es un material empleado en la técnica por deposición fundida en las impresoras 3D, la composición química $\left(\left(\mathrm{C}_{3} \mathrm{H}_{4} \mathrm{O}_{2}\right)_{\mathrm{n}}\right)$ de este material hace que sea ideal para ser sometido al proceso de conformación de muestras de diferentes geometrías en las impresoras 3D a temperaturas de hasta $159^{\circ} \mathrm{C}$, utilizando principalmente coordenadas tridimensionales. Las propiedades mecánicas de este material son: módulo de Young de entre 3.3 hasta 3.6 $\mathrm{GPa}$, un coeficiente de Poisson de entre 0.38 a 0.4 destacando su resistencia a la compresión de entre 66 hasta $86 \mathrm{MPa}$. A su vez existen estudios que demuestran que a partir del patrón generado en la impresión 3D, el objeto creado puede fallar en puntos específicos dependiendo de su geometría [1-2-34].

Por otra parte, existen estudios como el procedimiento eléctrico para la medición de la elongación con la ayuda de galgas extensométricas para determinar de forma indirecta las tensiones reales en la superficie de un material. Las galgas extensiométricas se componen de alambres (resistencias) que se adhieren a la superficie de una muestra. De esta forma, si la superficie sufre una elongación, el alambre se alarga y su sección transversal aumenta incrementando la resistencia 
eléctrica; si se produce una compresión sobre el material, la resistencia en la galga extensiométrica disminuye. El circuito empleado por las galgas extensiométricas, es un puente de Wheatstone y las resistencias se conectan como divisores de tensión. Esta conexión de medición, es especialmente apropiada para la medición de pequeños cambios en la superficie del material (elongación) por una perturbación, y por lo tanto, la determinación del cambio de resistencia de una galga extensiométrica [5-6-7].

La correlación de imagen digital (DIC) por técnica de moteado, es un método óptico sin contacto para medir y evaluar las coordenadas tridimensionales de superficies 3D en cuanto a elongación y deformación. Durante este proceso, se utilizan patrones/puntos de referencia para describir áreas de identificación sobre la superficie del material. Las áreas, se pueden analizar con una precisión de sub-píxel mediante el análisis de la información de la imagen. Finalmente, los resultados de medición del área de estudio del material, determinar el comportamiento estático y dinámico de las muestras, con el propósito de establecer una predicción de donde la muestra puede fallar. Por lo tanto, la técnica de correlación de imagen digital, permite llevar a cabo una investigación de tipo analítica y de esta forma evaluar el comportamiento de cualquier tipo de geometría a escala [8$11]$.

El patrón de moteado es generado por la interferencia aleatoria de luz esparcida desde varios puntos ubicados en la superficie del objeto de estudio, esta luz actúa como una rejilla natural impresa en el objeto para su análisis en la superficie, cuando se aplican esfuerzos sobre la muestra. Se han reportado varios estudios utilizando este método para medir deformaciones en la superficie de los materiales. El efecto de moteado puede usarse para obtener información de los desplazamientos a lo largo de los ejes $\mathrm{x}, \mathrm{y}, \mathrm{z}$ de un sistema de coordenadas previamente establecido sobre la superficie de la muestra. Por lo tanto, se debe tener en consideración la dirección de iluminación y el campo visual de estudio, pueden crearse sistemas experimentales sensibles en la muestra y en los alrededores de la misma [8].

GOM-Correlate ${ }^{\circledR}$ versión estudiantil, es la herramienta computacional que se emplea para el análisis de esfuerzos. Inicialmente, se considera el objeto que se quiere analizar, como el programa de cómputo efectúa la correlación de imágenes a partir de un aspecto específico en la muestra basado en patrones estocásticos, es indispensable que la muestra se encuentre en condiciones superficiales adecuadas para efectuar el análisis.

Existen dos formas de obtener la correlación de imagen digital cuando la muestra se encuentra sujeta al efecto mecánico de interés. Una forma, es a partir de una ráfaga de imágenes que generan una secuencia cuando se está aplicando un esfuerzo, y la otra forma, es a través de un registro por medio de video que captura la perturbación sobre la muestra. De esta forma, se deben tener en cuenta las siguientes consideraciones, la opción de registro con video no debe exceder 25 s por cuestion de recursos computacionales.

En la presente investigación se llevó a cabo el análisis mecánico del material PLA con el uso del software GOMCorrelate ${ }^{\circledR}$ para determinar el máximo esfuerzo generado durante la prueba de compresión. Durante la prueba, se utilizó la simulación numérica para establecer la relación éntrelos resultados experimentales obtenidos directamente del análisis de esfuerzos por el método de correlación de imágenes y con el uso de análisis numérico utilizando el software Solidworks.

\section{Materiales y métodos}

\subsection{Obtención de la muestra.}

La muestra utilizada para las pruebas, fue dibujada en un programa computacional CAD (Solidworks) con $110 \mathrm{~mm} \times$ $55 \mathrm{~mm} \times 10 \mathrm{~mm}$, como se muestra en la figura 1 .

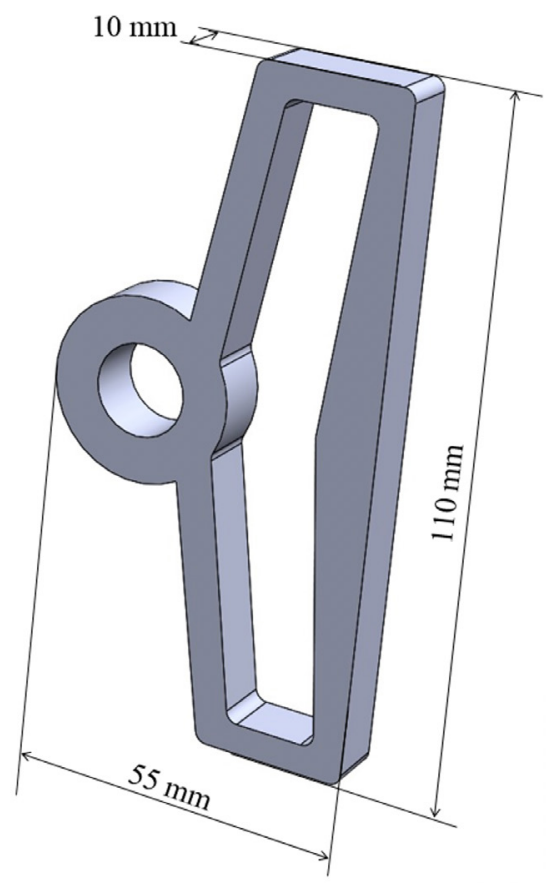

Figura 1. Geometría y dimensiones de muestra en CAD.

Una vez que se obtuvo el modelado tridimensional de la muestra utilizando una impresora $3 \mathrm{D}$, se empleó la técnica por deposición fundida para obtener un modelo tangible del material PLA, adquirir las muestras y efectuar los ensayos experimentales. Las principales propiedades mecánicas del PLA se presentan en la tabla 1: 
Tabla 1. Principales propiedades mecánicas del PLA.

\begin{tabular}{ll}
\hline \multicolumn{1}{c}{ Material } & \multicolumn{1}{c}{ PLA } \\
\hline Temperatura de fusión $\left({ }^{\circ} \mathrm{C}\right)$ & $145-177$ \\
Densidad $\left(\mathrm{kg} / \mathrm{m}^{3}\right)$ & $1.24 \times 10^{-3}$ \\
Dureza $(\mathrm{MPa})$ & $166-264$ \\
Módulo de Young $(\mathrm{MPa})$ & $330-360$ \\
Resistencia a compresión $(\mathrm{MPa})$ & $66-86$ \\
Limite elástico (MPa) & $55-72$ \\
Coeficiente de Poisson & $0.38-0.40$ \\
\hline
\end{tabular}

Fuente: [9]

\subsection{Prueba de compresión.}

El efecto mecánico generado en la muestra, es el de compresión y consiste en el esfuerzo al que está sometido un cuerpo por la aplicación de una fuerza por longitud de área, las cuales se ejercen en el mismo sentido y tienden a desplazar el cuerpo de sus coordenadas iniciales. Para calcular el acortamiento de una muestra, se emplea la ecuación 1:

$$
\delta=\frac{\mathrm{F} \times \mathrm{L}}{\mathrm{E} \times \mathrm{A}}
$$

Dónde: $\delta$ es el alargamiento $(\mathrm{cm}), \mathrm{F}$ es la fuerza de tracción en $\mathrm{KgF}$, L es la longitud de la muestra $(\mathrm{cm})$, E es el módulo de elasticidad del material $\left(\mathrm{KgF} / \mathrm{cm}^{2}\right)$ y A es la sección transversal $\left(\mathrm{cm}^{2}\right)$.

Teniendo en cuenta lo anterior, la muestra se preparó utilizando el siguiente procedimiento:

1. Se cubre en su totalidad de color blanco.

2. Se realiza un moteado en color negro, como sugerencia el moteado en negro debe ser aplicado a $45^{\circ}$ y a una distancia de entre $20 \mathrm{~cm}$ y $30 \mathrm{~cm}$ de la muestra con el propósito de no cubrir zonas completamente en la muestra de negro.

3. Finalmente, se generan puntos minúsculos en la muestra que funcionan como patrones de identificación.

La finalidad del moteado, es que se detecten los patrones sobre la muestra y sea posible analizarla con el software GOM-Correlate ${ }^{\circledR}$. Se tomaron videos de entre 20 s y 25 s para captar el efecto mecánico en la muestra, siguiendo el procedimiento descrito anteriormente, y de esta forma, no demandar mucho recurso computacional y sean tiempos cortos de análisis. En la figura 2 se observa a la muestra bajo la técnica de moteado para realizar análisis respectivos.

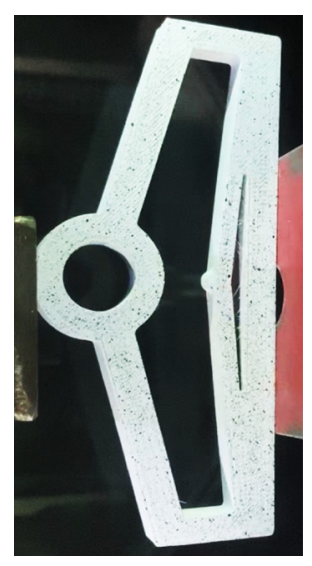

Figura 2. Técnica de moteo en muestra de estudio.

El software GOM Correlate ${ }^{\circledR}$ es utilizado para realizar el análisis de imagen directamente del registro de video durante la prueba de compresión, el cual, realiza el mapeo de imagen; este mapeo de imágenes actúa como una interfaz de usuario intuitiva que apoya al usuario en todos los pasos de trabajo para la definición del proyecto, el análisis de los resultados y los informes. La figura 3 presenta un diagrama de la serie de pasos para obtener el análisis de esfuerzos con la técnica de la DIC.

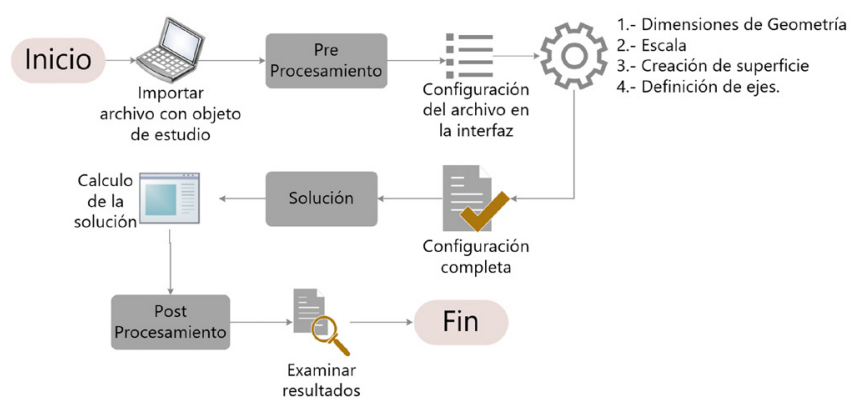

Figura 3. Procedimiento para evaluación de la muestra mediante la DIC

Todas las interacciones necesarias del usuario, se realizan directamente en las imágenes de medición y los resultados se muestran instantáneamente como campos vectoriales, de desplazamiento y de tensión de campo completo sobre la superficie de la muestra.

Posteriormente, se importa el archivo contenido de registro de video y automáticamente el programa lo distribuye en imágenes secuenciales en una línea de tiempo que debe ser ajustado por el usuario. Finalmente, se comparan los parámetros de medida de la muestra que arroja el programa y de ser necesario, se modifican a la medida original de la muestra y se define una escala de trabajo. Luego, se genera una superficie de respuesta de identificación del patrón y el programa automáticamente detecta una serie de puntos que puede analizar. El usuario tiene la ventaja de crear la 
superficie en el área que sea de interés, con esto se pueden reducir puntos de análisis innecesarios y concentrar el estudio en zonas de importancia. Una vez establecida la zona de trabajo, se procede a seleccionar en que eje del plano 2D se ve afectada a la muestra, para este objeto en particular, el eje de estudio es el de las abscisas debido a que la compresión en la muestra es de forma horizontal. Así que se selecciona la opción de "Épsilon (X)" como forma de identificación para el software GOM-Correlate ${ }^{\circledR}$.

Automáticamente la plataforma despliega una barra de colores, la cual cambia en relación al movimiento de la línea de tiempo generada para la muestra. Por otra parte, se identificó en la superficie de estudio que existe un cambio de deformación considerable, y con ayuda de la gráfica de colores es posible determinar el valor y porcentaje de deformación en el punto específico sobre la superficie.

\subsection{Simulación numérica}

Un método de comparación para en análisis de estudio en la presente investigación consistió en la construcción de una simulación por medio del software de cómputo SolidWorks ${ }^{\circledR}$ versión estudiantil. Empleando la herramienta de asistente al usuario, para análisis de esfuerzos se analizan los desplazamientos y la tensión de Von Mises cuando se aplica un esfuerzo de compresión sobre la muestra debido a la aplicación de la carga.

\section{Resultados y discusiones}

\subsection{Resultados de la prueba de compresión utilizada el} software GOM Correlate ${ }^{\circledR}$.

Las cargas aplicadas a la muestra comienzan desde $0 \mathrm{~N}$ hasta $1 \mathrm{~N}$, con dos esfuerzos importantes: $0.5 \mathrm{~N}$ y $1 \mathrm{~N}$. A través de la línea de tiempo, se observa la compresión de la muestra y como se desplaza el material hacia el punto de sujeción.

Con la finalidad de obtener resultados del desplazamiento sobre la muestra, se seleccionó la zona de trabajo en la cual la paquetería de cómputo interpreta como coordenadas. Así que, los resultados están asociados específicamente a la muestra de estudio. Durante este proceso se empleó una maquina para ensayos de compresión, el cual consiste en la aplicación de torque y una fuerza de forma manual en donde la muestra de PLA fue ensayada para determinar el desplazamiento utilizado del programa de computo considerado para este estudio experimental. Para accionar la maquina de ensayos se gira una perilla que acciona un tornillo sin fin generando esfuerzos de compresión entre dos placas. Es importante considerar la calibración del instrumento para evitar mediciones erradas.

La figura 4 presenta la muestra en estado de reposo $(0 \mathrm{~N})$, se observa que en la superficie de la muestra el software creo un área de color para la identificación de eje (Épsilon X) y el porcentaje de desplazamiento en la muestra se mantiene en 0 .

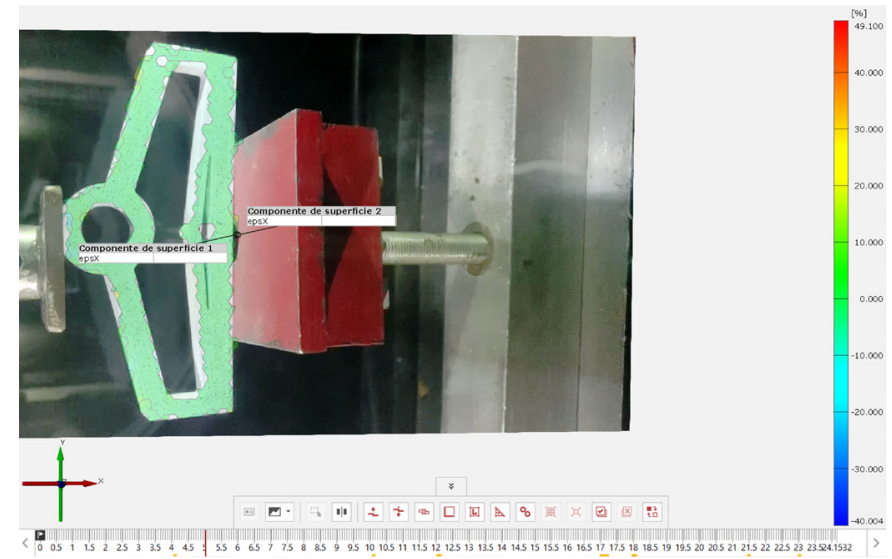

Figura 4. Muestra en estado de reposo (0 N y 5 s).

Posteriormente, se acciono el mecanismo de compresión sobre la muestra exactamente en el orificio superior, el valor de carga inicial registrado por el aparato de ensayo es de 0.3 N.

En lafigura 5 se observa que la muestra presenta un cambio en la geometría debido a la carga ejercida, el porcentaje de desplazamiento se encuentra entre $0 \%$ y $20.000 \%$, lo cual, significa que la muestra sufrió un porcentaje significativo de desplazamiento con la aplicación de un esfuerzo.

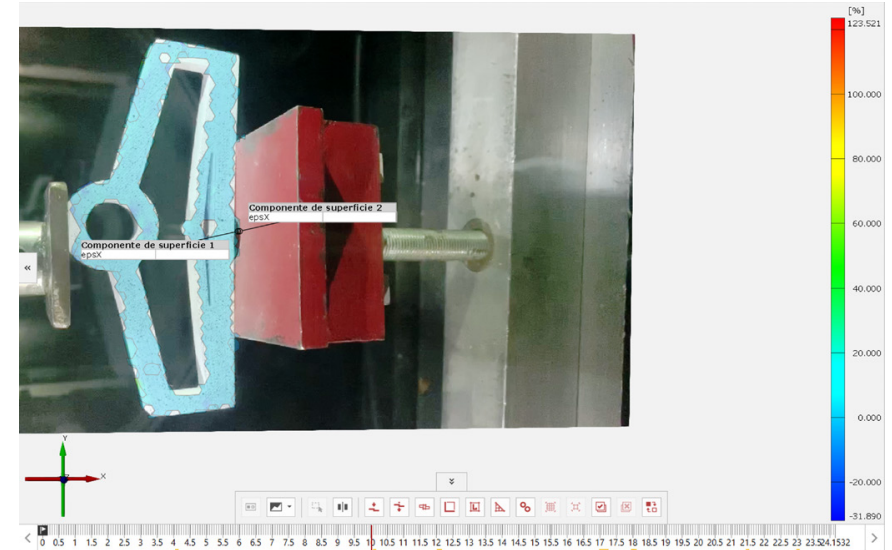

Figura 5. Aplicación de $0.5 \mathrm{~N}$ sobre la muestra de estudio en 10

Conforme continúa el análisis, en la figura 6 se acciono la maquina de ensayo nuevamente hasta llegar a una carga aplicada de $1 \mathrm{~N}$. De esta forma, el máximo de esfuerzo de compresión aplicado en la muestra está relacionado con las propiedades mecánicas del material (PLA). 


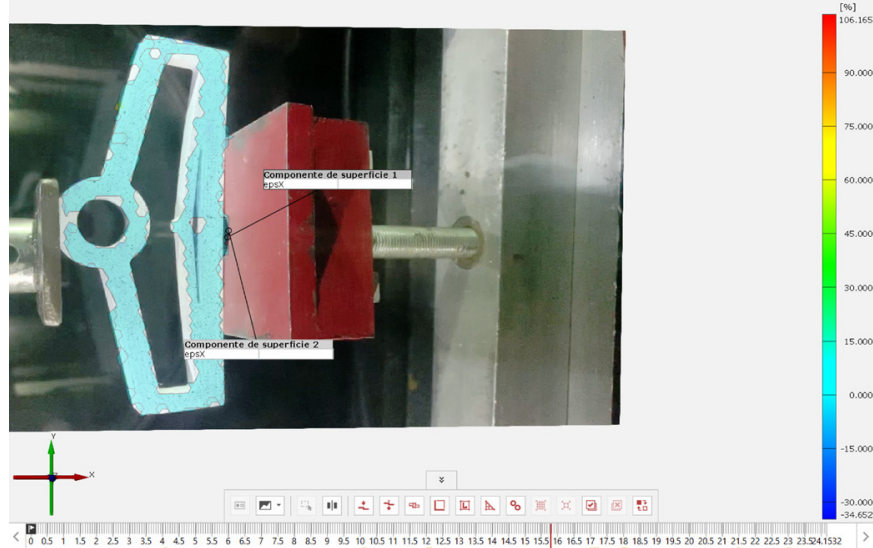

Figura 6. Aplicación de carga máxima (1 N) en 15.725 s.

El ensayo experimental en apoyo de la paquetería de cómputo seleccionada revela que los desplazamientos en la muestra alcanzan hasta un máximo de $15 \%$ de desplazamiento en toda la muestra, para las condiciones experimentales utilizadas. Finalmente, la descarga total una vez finalizada la se presenta, se muestra en la figura 7, donde el porcentaje de desplazamiento en la barra de colores vuelve otra vez al valor de $0 \%$ de desplazamiento, siendo la forma original del material dado a que la muestra no excedió el esfuerzo de fluencia del material.

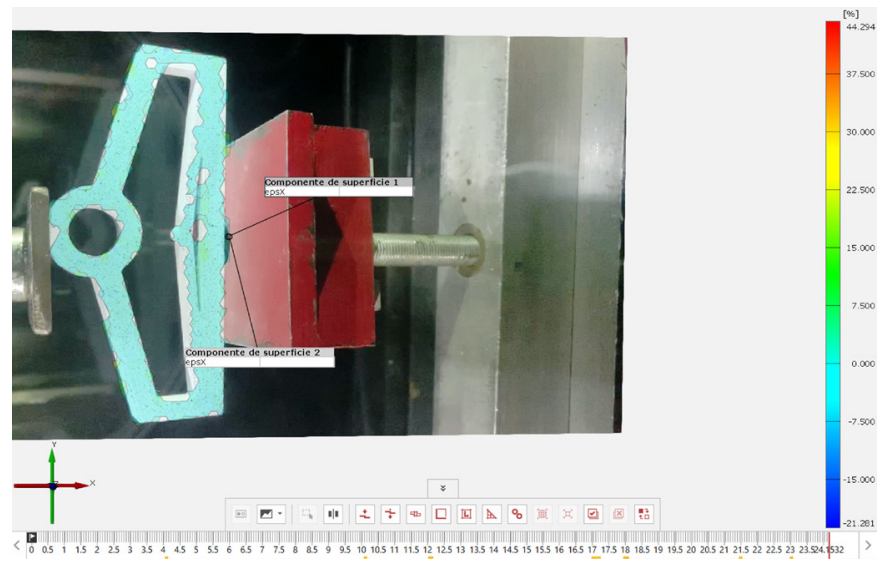

Figura 7. Descarga total del esfuerzo aplicado en la muestra (24.1532 s).

3.2 Resultados de simulación de la prueba de compresión. Estableciendo las condiciones del efecto de compresión aplicado en la máquina de ensayos, se seleccionó la cara inferior de la muestra como una cara fija, es decir, sin desplazamiento. Por otro lado, en la parte superior de muestra en estudio, en la geometría circular se aplicó un esfuerzo de hasta $1 \mathrm{~N}$ aplicando un esfuerzo de compresión sobre la muestra desde la parte superior hasta la inferior.

Los resultados del análisis de esfuerzos que arroja la paquetería de cómputo son: la trama de desplazamiento estático (URES) y la tensión de von Mises. Los resultados demuestran que la principal deformación de la muestra se encuentra en la parte superior, mientras que la parte inferior presenta valores despreciables por la compresión efectuada. La figura 8 presenta los valores sobre el desplazamiento estático en la muestra con un valor de $1 \times 10^{30}$ siendo despreciables para las condiciones experimentales utilizadas durante las prueba, por otra parte, el máximo del esfuerzo presento un valor de $3.686 \times 10-3 \mathrm{~mm}$, donde no alcanza los valores de resistencia a la compresión máximos del material [10-12-13-14]. Bajo estas condiciones experimentales, el material no excede la zona elástica del material, y por lo tanto, el material recupera su condición inicial (ver Tabla 1).

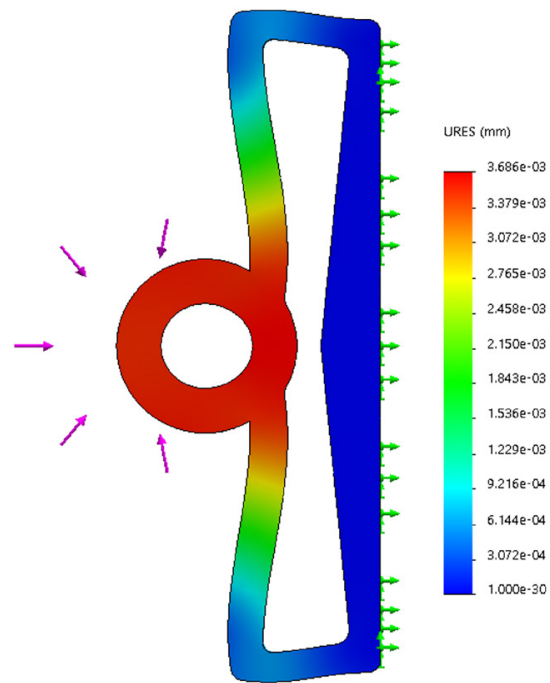

Figura 8. Trama de desplazamiento estático por compresión de $1 \mathrm{~N}$.

Por otra parte, la figura 9 presenta los resultados de la tensión de von mises la cual representa la magnitud física proporcional a la energía de distorsión, es decir, valores mínimos y máximos de entre $1.355 \times 10^{2}$ y $8.131 \times 10^{2} \mathrm{MPa}$, respectivamente [15-16-17].

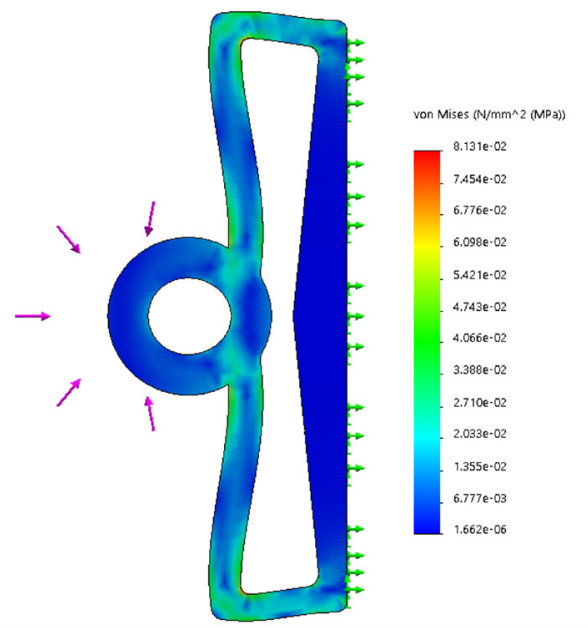

Figura 9. Tensión de von Mises por compresión de 1 N. 


\section{Conclusiones}

El empleo de herramientas computacionales especializadas por correlación de imagen digital resulta ser de gran utilidad ya que permiten predecir el nivel de deformación que experimenta una muestra a determinados esfuerzos. Hoy en día el buen desempeño de un elemento mecánico en el área industrial es vital ya que evita accidentes mortales y la interrupción de actividades por falla.

La preparación de materiales para emplear en GOMCorrelate ${ }^{\circledR}$ es sencilla, pero requiere detallar con muy bien el patrón estocástico de identificación, con los resultados obtenidos se puede establecer una comparación frente a otros métodos de cálculo de deformación en materiales

Mediante los análisis experimentales y de simulación, se logró validar que el material soporta las cargas aplicadas sin exceder el límite elástico del material. De esta manera, se validaron los esfuerzos generados sobre la muestra bajo cargas normales.

El material bajo condiciones ideales superara el esfuerzo de fluencia, cuando la aplicación de la carga sobrepase $\sim 55$ MPa durante la ejecución de la prueba, iniciando con el modo de falla de agrietamiento por fatiga.

\section{Referencias}

[1] X. Li, Z. Ni, S. Bai y B. Lou. Preparation and Mechanical Properties of Fiber Reinforced. IOP Conference Series: Materials Science and Engineering, 2018.

[2] O. Martin y L. Averous. (Polylactic acid): pasticization and properties of biodegradable miltiphase systems. POLYMER, no. 42, pp. 62096219, 2001.

[3] F. Senatov, K. Niaza, M. Zadorozhnyy, A. Maksimkin, S. Kaloshkin y Y. Estrin. Mechanical properties and shape memory effect. Journal of the mechanical behavior of biomedical materials, no. 57, pp. 139-148, 2015.

Doi: $\quad$ https://doi.org/10.1016/j.jmbbm.2015.11.036

[4] B. Steve y D. Rickerby, Handbook of hard coatings, de Deposition technologies, properties and applications, Noyes Publication, 2001.

[5] M. Fitzpatrick, A. T. Try, P. Holdway, F. Kandil, J. Shackleton y L. Suominen, Determination of residual stress by X-Ray diffraction, National Physical Laboratory, pp. 1-68, 2005.

[6] G. Rodríguez-Rodríguez, A. Hernández-González, y A. López-Llanusa. Sistema para la medición de deformación. Revista de Ingeniería Electrónica, Automática y Comunicaciones, vol. 37, no. 1, pp. 80-93, 2016.

[7] E. Alzate-Rodríguez, J. Montes-Ocampo y C.
Silva-Ortega. Medidores de deformacion por resistencia: galgas extensiométricas. Scientia et Technica, vol. 34, no. 8, pp. 7-12, 2007. Doi: https://doi.org/10.22517/23447214.5519

[8] N. Alcalá-Ochoa, R. Rodríguez-Veral y B. Barrientos, Técnica de moteado para medición de fracturas en estructuras metálicas, Revista Mexicana de Física, vol. 46, no. 5, pp. 468-477, 2000.

[9] M. Foruzanmehr, P. Vuillaume, S. Elkoun y M. Robert. Physical and mechanical properties of PLA composites reinforced by $\mathrm{TiO} 2$ grafted flax fibers. Materials and Design, no. 106, pp. 295304, 2016. Doi: https://doi.org/10.1016/j.matdes.2016.05.103

[10] J. Resendiz-Hernández y L. Palacios- Pineda. Análisis de deformaciones unitarias en juntas soldadas utilizando correlación digital de imágenes. Revista de Ingeniería Mecánica, vol. 1, no. 1, pp. 10-19, 2017.

[11] T. Chuen-Lin y Z. Hung-Da, Measuring residual stress of anisotropic thin film, optics express, vol. 18, no. 16, pp. 1-7, 2010.

[12] M. Dos-Santos-Ayres, A. Vogel, J. Duarte, H. Reinert y F. Detke, Validación de deformaciones por técnica de correlación de imágenes digitales. Salo do conhecimiento, pp. 1-5, 2016.

[13] N. López y R. H. I. Ygel. Análisis de una junta experimental de columna y vigas de concreto armado-columna de acero para pórticos utilizando correlación de imágenes digitales. Revista Gaceta Técnica, vol. 19, no. 1, pp. 25-42, 2018.

[14] R. A. García-León, A. Bohorquez-Niño, and J. F. Barbosa-Paredes. Design of an extrusion machine for the manufacture of plastic tubes. J. Phys. Conf. Ser., vol. 1257, p. 12006, 2019.

[15] M. Á. Caminero, J. M. Chacón, E. García-Plaza, P. J. Núñez, J. M. Reverte, and J. P. Becar, "Additive manufacturing of PLA-based composites using fused filament fabrication: Effect of graphene nanoplatelet reinforcement on mechanical properties, dimensional accuracy and texture," Polymers (Basel)., vol. 11, no. 5, 2019. Doi: 10.3390/ polym11050799

[16] A. Rigoussen, J.-M. Raquez, P. Dubois, and P. Verge, "A dual approach to compatibilize PLA/ABS immiscible blends with epoxidized cardanol derivatives," Eur. Polym. J., vol. 114, pp. 118-126, 2019.Doi: https://doi.org/10.1016/j. eurpolymj.2019.02.017

[17] E. O. Cisneros-López et al., "Recycled poly(lactic acid)-based 3D printed sustainable biocomposites: a comparative study with injection molding," Mater. Today Sustain, vol. 7-8, p. 100027, 2020. Doi: https://doi.org/10.1016/j.mtsust.2019.100027 